\title{
Percepções Infantis: Relações entre Motivação Escolar e Suporte Familiar
}

\author{
Andréia Arruda Guidetti - Universidade Paulista, São Paulo, Brasil \\ Selma de Cássia Martinelli - Universidade de Campinas (UNICAMP), Campinas, Brasil
}

\begin{abstract}
Resumo
Esta pesquisa teve por objetivo analisar a percepção infantil da motivação escolar e do suporte familiar dos alunos, de diferentes anos escolares, e verificar as relações entre as variáveis. Participaram 342 crianças, de ambos os sexos, com idades de sete a 13 anos, de três escolas municipais do interior do estado de São Paulo. Foram investigadas as orientações motivacionais e a percepção infantil do suporte familiar. Os resultados revelam que as orientações motivacionais e o suporte educativo familiar apresentaram declínio conforme o avanço da escolaridade, houve correlação positiva, significante e baixa entre a motivação intrínseca e o suporte familiar afetivo, educativo e material, e correlação negativa, significante e baixa entre a motivação extrínseca e o aspecto afetivo do suporte familiar. Conclui-se pela importância do aprofundamento dos conhecimentos do suporte familiar na motivação escolar, visto que este se mostra relacionado à motivação intrínseca dos alunos com o progredir da escolaridade.

Palavras-chave: ensino fundamental, avaliação, suporte familiar, motivação escolar
\end{abstract}

Infant Perceptions: Relationships between School Motivation and Family Support

\begin{abstract}
The aim of this research was to analyze children's perception of school motivation and the family support of students from different school years, as well as verifying the relations between variables. This study was composed of 342 children, with ages ranging from 7 to 13 , both male and female, from three municipal schools in the countryside of the state of São Paulo. We investigated the motivational orientations and children's perception of family support. The results indicate that the motivational orientations and the family educational support showed a decline as school years progresses; there was positive, meaningful and low correlation between the intrinsic motivation and the affective, educational and material family support; and negative, meaningful and low correlation between the extrinsic motivation and the affective aspect of the family support. This study concluded the importance of deepening the knowledge of family support in the school motivation, since this is shown as related to the students' intrinsic motivation with the schooling progress.

Keywords: elementary school, evaluation, family support, school motivation
\end{abstract}

Percepciones Infantiles: las Relaciones entre la Motivación Escolar y el Soporte Familiar

\section{Resumen}

Esta investigación tuvo por objetivo analizar la percepción infantil entre la motivación escolar y el soporte familiar de los alumnos de diferentes años escolares, y también verificar las relaciones entre las variables. Participaron del estudio 342 alumnos de tres escuelas municipales del interior del Estado de San Pablo, de ambos sexos con edades entre 7 a 13 años. Fueron investigadas las orientaciones motivacionales y la percepción infantil del soporte familiar. Los resultados muestran que las orientaciones motivacionales y el soporte educativo familiar van declinando de acuerdo con el avance de la escolaridad, hubo correlación positiva, significativa y baja entre la motivación intrínseca y el soporte familiar afectivo, educativo y material, y correlación negativa, significativa y baja entre la motivación extrínseca y el aspecto afectivo del soporte familiar. Se concluye con la importancia de profundizar los conocimientos del soporte familiar en la motivación escolar, ya que esto se muestra relacionado con la motivación intrínseca de los alumnos con el progreso de la escolaridad.

Palabras-clave: Enseñanza Primaria; evaluación; soporte familiar; motivación escolar

O desenvolvimento humano é continuamente influenciado por diferentes aspectos, sejam eles cognitivos, sociais ou afetivos e, em cada fase da vida, os indivíduos contam com sistemas de suporte diferenciados para enfrentar as adversidades impostas pelo desenvolvimento. No período escolar, as relações de envolvimento entre família e escola surgem como indicadores de suporte necessários tanto para o desenvolvimento quanto para as aprendizagens infantis.
Manter-se motivado a prosseguir e enfrentar as dificuldades que aparecem no decorrer do processo escolar é apenas um dos desafios a ser enfrentado. À motivação tem sido dada grande atenção, visto ser considerada de fundamental importância na vida dos indivíduos e no desempenho escolar dos alunos (Martinelli \& Sisto, 2011). As condições que podem influenciar essa motivação são diversas, podendo ser considerados desde os fatores internos ao indivíduo e o contexto escolar 
(Martinelli \& Sisto, 2011) até o meio familiar (Guidetti, 2013).

Neste estudo, priorizou-se olhar para a motivação e para o suporte familiar. De acordo com Ryan, Lynch, Vansteenkiste e Deci (2011), a motivação implica a energia e a direção da ação e pode ser aplicada em todos os contextos de atuação do ser humano, mas o objetivo deste trabalho é explorá-la no contexto educacional, pois se o motivo é aquilo que move uma pessoa ou que a põe em ação para atingir um objetivo e finalizá-lo, indiscutivelmente, é primordial nas ações educacionais.

Tendo como base a Teoria da Autodeterminação (TAD), este estudo priorizou a investigação da orientação motivacional intrínseca e extrínseca do estudante. A TAD, proposta por Deci e Ryan, na década de 1970 do século XX, focaliza as tendências naturais humanas para o crescimento, desenvolvimento e bem-estar no processo de interação com as condições socioculturais. $\mathrm{Na}$ TAD, pode-se distinguir entre diferentes tipos de motivação, baseados nos diferentes motivos ou objetivos que dão origem a uma ação, e a distinção mais básica é entre a motivação intrínseca, que se refere a fazer algo porque é intrinsecamente interessante ou agradável, e a motivação extrínseca, que se refere a fazer algo porque isso leva a um resultado externo (Ryan \& Deci, 2000). De acordo com a TAD (Deci \& Ryan, 2008), os comportamentos humanos baseiam-se na satisfação de necessidades psicológicas fundamentais de autonomia, competência e pertencimento.

A necessidade de autonomia está vinculada ao desejo de se autogovernar, organizar sua própria experiência e comportamento e integrá-los ao seu self. A competência é a capacidade do organismo de interagir satisfatoriamente com o seu meio, o que favoreceria o desenvolvimento e a aprendizagem de algumas capacidades, e o pertencimento está ligado ao fato de o ser humano necessitar manter vínculos emocionais e interpessoais com pessoas significativas (Deci \& Ryan, 2008). As necessidades de autonomia, competência e de pertencimento são integradas e interdependentes, o que faz com que o fortalecimento ou satisfação de uma reforce às demais, e são essenciais para a motivação intrínseca, que compreende crescimento, integridade e coesão social do indivíduo que realiza determinada atividade por ser de alguma forma geradora de satisfação e, sobretudo, com ausência de constrangimentos externos ou internos (Martinelli \& Sisto, 2011).

No sentido oposto da orientação motivacional intrínseca está a extrínseca, que busca atender a pressões e comandos de outras pessoas com o intuito de demonstrar competências e habilidades. Baseados na TAD, Próspero, Russel e Gupta (2012) afirmam que a motivação extrínseca está relacionada à satisfação de necessidades de autoestima e que a maioria das atividades desempenhadas pelas pessoas é extrinsecamente motivada, especialmente na infância, quando a liberdade de escolha está cerceada por funções que exigem dos indivíduos realizar tarefas e demandas sociais impostas. Embora comportamentos extrinsecamente motivados não sejam inerentemente interessantes, pois são controlados por recompensas e ameaças com vista a um fim que não o próprio prazer em fazer algo e diminuem caso as recompensas ou pressões externas sejam retiradas, são frequentemente solicitados e, de maneira geral, o motivo que leva os alunos a responderem a essas solicitações são as avaliações das pessoas importantes de seu contexto, como professores, familiares, amigos, entre outros (Ryan \& Deci, 2000).

Ao discorrer sobre a motivação e o desempenho escolar, Martinelli e Sisto (2011) ressaltam que a conduta do aluno geralmente é motivada conjuntamente por motivos intrínsecos e extrínsecos, embora uma ou outra forma de motivação possa prevalecer. Os autores enfatizam que muitos conteúdos ou destrezas são importantes do ponto de vista acadêmico e social, contudo, não apresentam um interesse intrínseco, e que, nesse caso, necessitariam de princípios extrínsecos para serem eliciados. Diante dessas informações, e ao considerar que a motivação pode influenciar no modo como o aluno utiliza suas capacidades, podendo afetar seu desempenho escolar, acredita-se que a qualidade do desempenho e da aprendizagem de um aluno se diferenciará à medida que este for intrínseca ou extrinsecamente motivado, como afirmam Martinelli e Sisto (2011).

Desse modo, ao se pautar na TAD, que é a base teórica desta pesquisa, estudos nacionais e internacionais têm evidenciado a prevalência da motivação intrínseca nos estudantes da etapa inicial do Ensino Fundamental, indicando que essa motivação tende a diminuir com o decorrer das séries cursadas, dando lugar à motivação extrínseca, que costuma vigorar principalmente na segunda etapa do Ensino Fundamental, que compreende do $6^{\circ}$ ao $9^{\circ}$ ano. Revelam, ainda, uma diminuição gradativa tanto da motivação intrínseca quanto da extrínseca à medida que os alunos avançavam nas séries escolares (Ferreira, Cardoso, \& Abrantes, 2011; Gillet, Vallerand, \& Lafrenière, 2012; Guidetti, 2013; Hornstra, Veen, Peetsma, \& Volman, 2013; Lemos \& 
Veríssimo, 2014; Martinelli \& Sisto, 2010; Meyersa, Pignaultb, \& Houssemandc, 2013).

Assim como a motivação, o suporte familiar, de acordo com a literatura, é um construto importante quando se estuda a criança no seu percurso inicial de escolarização. De acordo com Polonia e Dessen (2005), a família e a escola emergem como duas instituições fundamentais para desencadear os processos evolutivos das pessoas, atuando como propulsores ou inibidores do seu crescimento físico, intelectual e social. Como nos demais aspectos do desenvolvimento infantil, a importância da participação da família no processo de aprendizagem é inegável.

Os estudos de Andrada (2007), Horn et al. (2009), Monteiro (2010) e Bradley, McKelvey e Mansell (2011) investigam o suporte familiar afetivo e educativo relacionado ao contexto escolar. Os autores discutem que o suporte familiar para a realização escolar se caracteriza pelo envolvimento dos pais na vida acadêmica dos filhos, definindo horários e supervisionando o estudo e as tarefas, mantendo contato regular com professores e estando disponíveis para ajudar, se necessário. Destacam ainda a importância do engajamento dos pais em atividades diversas da vida infantil, incluindo o tempo que passam com as crianças, a qualidade das interações familiares e a resposta emocional dos pais ante as demandas dos filhos.

Por sua vez, Guidetti (2007), Marturano (2006), e Bradley et al. (2011) aventam que o suporte material da família, definido de acordo com a renda familiar, e as variáveis de status socioeconômico, como moradia, alimentação e vestuário, além da disponibilidade de materiais, livros e brinquedos educativos, são dimensões importantes que podem influenciar diretamente o desempenho acadêmico infantil. De acordo com Guidetti (2013), não se pode desconsiderar que os recursos materiais da família são indispensáveis, mas é na relação entre os membros que esses recursos podem ser utilizados de forma a enriquecer o desenvolvimento global do aluno e, especificamente, o seu desempenho escolar.

Nesse sentido, a concepção utilizada nessa investigação entende o conceito de suporte familiar voltado para as questões escolares como um construto multifacetado, que engloba o envolvimento parental nos aspectos afetivos, educativos e materiais. $\mathrm{O}$ aspecto afetivo compreende a dimensão subjetiva, isto é, os pensamentos e sentimentos infantis expressos na relação com os pais, como sentir-se amado por eles, receber elogio pelo bom desempenho escolar e participar da vida familiar. Já o aspecto educativo pode ser considerado como a organização da rotina diária infantil, em diferentes momentos, como fazer a lição ou ter horários definidos para dormir e fazer as refeições. Por último, o aspecto material enfatiza os recursos materiais e físicos disponíveis no ambiente familiar, como livros, brinquedos, materiais escolares e espaço para a realização das atividades escolares.

Uma revisão bibliográfica sobre o que tem sido pesquisado em relação à temática permitiu observar os estudos nacionais (Andrada, 2007; Cia, Pamplin, \& Williams, 2008; Guidetti, 2013; Guidetti \& Martinelli, 2009) e internacionais (Aikens \& Barbarin, 2008; Cheung \& Pomerantz, 2011; Galindo \& Sheldon, 2012; Horn et al., 2009; Willems \& Gonzalez-DeHass, 2012; Yune, 2014) que têm se preocupado em discutir a relação entre suporte familiar e desempenho. Essas pesquisas têm como participantes crianças do Ensino Fundamental, mas as investigações sobre o suporte familiar foram realizadas com os pais, ou seja, as percepções são do adulto. Esses estudos indicam que o envolvimento da família com a escolarização da criança aumenta suas conquistas acadêmicas, e os benefícios são evidenciados em maiores pontuações em testes, melhores notas e atitudes mais positivas sobre os trabalhos escolares.

A quantidade de trabalhos internacionais que trataram dessa questão é mais acentuada, ainda que não seja muito extensa, entretanto, os estudos têm priorizado a percepção do adolescente sobre os suportes familiares e a motivação escolar (Bong, 2008; Iachini, Buettner, Anderson, \& Reno, 2013; Moreira, Dias, Vaz, \& Vaz, 2013). Os resultados desses estudos mostram significativas correlações entre o ambiente familiar e a motivação dos alunos, ressaltando que os estudantes mais bem-sucedidos academicamente tendem a ter pais mais exigentes e que estão ativamente envolvidos na educação de seus filhos, sendo as interações familiares positivas na percepção dos filhos. Diante do panorama apresentado, observa-se que as pesquisas que investigam a relação entre motivação e suporte familiar foram realizadas, em sua grande maioria, utilizando a visão dos pais ou responsáveis ou, algumas vezes, de filhos adolescentes. $\mathrm{O}$ relato das crianças envolvidas nesse contexto tem sido pouco pesquisado.

Analisar a percepção infantil sobre o suporte familiar é um dos focos deste trabalho. Para Sternberg (2010), a percepção é o conjunto de processos psicológicos pelos quais é possível reconhecer, organizar, sintetizar e fornecer significação em nível cognitivo, as sensações provenientes dos estímulos ambientais. 


\section{Dessa forma, apresenta particularidades para cada} indivíduo, por isso a escolha da investigação voltada para a criança. Spera (2006) destaca que as percepções sobre os suportes parentais de crianças e adolescentes preveem melhores resultados do que a percepção dos pais, pois fornecem maiores detalhes de seus próprios pensamentos, emoções, motivações e comportamentos e de como eles ocorrem dentro do contexto familiar e se relacionam com o contexto escolar. Guidetti (2007) discorre sobre a importância da avaliação da percepção infantil e ressalta que, embora o suporte recebido e o percebido estejam diretamente ligados, a intensidade e a maneira como são sentidos pelos membros da família têm significados distintos. Assim, o objetivo do presente estudo é analisar dados referentes à percepção infantil da motivação escolar e do suporte familiar dos alunos, de diferentes anos escolares, e verificar as relações entre as variáveis.

\section{Método}

\section{Participantes}

Participaram desta pesquisa 342 crianças, regularmente matriculadas do $3^{\circ}$ ao $5^{\circ}$ ano do primeiro ciclo do Ensino Fundamental, com idades variando de sete a 13 anos e de ambos os sexos. Com relação à distribuição dos participantes por ano escolar, 84 (24,6\%) cursavam o $3^{\circ}$ ano, 134 (39,2\%), o $4^{\circ}$ ano e 124 (36,3\%) cursavam o $5^{\circ}$ ano. Esses participantes estavam regularmente matriculados em três escolas municipais situadas em uma cidade do interior do estado de São Paulo.

\section{Instrumentos}

Escala para Avaliação da Motivação Escolar - EAME-IJ (Martinelli \& Sisto, 2011). A escala tem como objetivo fornecer informações de como a criança e o adolescente se percebem em relação às fontes de motivação (intrínseca e extrínseca), bem como de sua intensidade. As categorias definidas para a motivação intrínseca foram: autodeterminação, competência, envolvimento na tarefa, curiosidade e interesse; e para a motivação extrínseca foram: preocupação com a avaliação, com o reconhecimento, com a competição, enfoque no dinheiro ou outro incentivo claro e enfoque na preocupação com os outros. A escala foi construída para aplicação em pessoas de oito a 11 anos e pode ser aplicada tanto individualmente quanto coletivamente.

Um primeiro estudo foi realizado com 367 participantes, com idades entre oito e 11 anos e que frequentavam de $2^{\mathrm{a}}$ a $4^{\mathrm{a}}$ série (ou $3^{\mathrm{o}}$ ao $5^{\mathrm{o}}$ ano). Com vistas a verificar a organização dos itens e a possibilidade de extração de fatores, procedeu-se à análise de adequação da amostra de Kaiser-Meyer-Olkin (KMO = $0,90)$ e o teste de esfericidade de Bartlett $\left(X^{2}=8219,76\right.$, $g l=1431, p<0,001)$, que indicaram a possibilidade de extração de mais de um fator para as 54 frases.

Os itens relacionados à motivação intrínseca formaram o fator 2 , e os itens concernentes à motivação extrínseca formaram o fator 1, explicando 43,86\% da variância. Além disso, os resultados do índice de Cronbach indicaram patamares de precisão bastante bons, tanto para a escala geral $(0,85)$ quanto para cada fator separadamente, fator $1(0,91)$ e fator $2(0,85)$. Em um segundo estudo, que contou com a participação de 862 alunos, que frequentavam oito diferentes escolas, de cinco cidades do interior do estado de São Paulo, de $2^{\mathrm{a}}$ a $8^{\mathrm{a}}$ série $\left(3^{\mathrm{o}}\right.$ ao $9^{\mathrm{o}}$ ano), com idades entre sete e 15 anos, reaplicou-se a escala constituída por 20 frases (10 concernentes a motivação intrínseca e outras 10 a motivação extrínseca). Procedeu-se novamente a análise de adequação da amostra de Kaiser-Meyer-Olkin (KMO = $0,90)$ e o teste de esfericidade de Bartlett $\left(X^{2}=4336,20\right.$, $g l=190, p<0,001)$, que mostrou novamente a configuração de dois fatores que explicaram $42,6 \%$ da variância, ficando mantida a estrutura anterior. A correlação entre os dois fatores foi de 0,005 , ou seja, nula, o que favoreceu a interpretação de independência deles.

Escala da Percepção Infantil dos Suportes do Ambiente Familiar - EPISAF (Guidetti \& Martinelli, 2010). Essa escala foi desenvolvida e construída a partir da literatura da área com base em instrumentos nacionais e internacionais (Baptista, 2005; Benetti \& Balbinotti, 2003; Dunst \& Leet, 1986; Fantuzzo, Tighe, McWaayne, Davis, \& Childs, 2002; Guidetti \& Martinelli, 2009; Marturano, 2006; Moos \& Moos, 1994). O objetivo da EPISAF é avaliar como a criança identifica o suporte familiar oferecido relativo a aspectos afetivos, educativos e materiais disponíveis no seu ambiente familiar.

A escala é composta por dimensões do suporte afetivo, que contemplam 14 itens, e suporte educativo, seis itens, e são avaliados por meio de quatro alternativas de respostas: "sempre", "muitas vezes", "poucas vezes", "nunca". O suporte material é avaliado por um questionário com 10 itens com a opção de resposta "sim" ou "não". Quanto maior é a pontuação da criança na escala, maior é sua percepção de suporte familiar.

Um estudo foi realizado com 306 participantes, com idades entre sete e 17 anos que frequentavam do $3^{\circ}$ ao $8^{\circ}$ ano. Os dados psicométricos dessa escala 
indicaram uma medida de adequação da amostra de Kaiser-Meyer-Olkin $(\mathrm{KMO}=0,87)$, julgando-se mais pertinente a utilização de uma rotação varimax; o método de extração dos fatores aplicado foi o de análise de componentes principais. Considerando que se desejava uma escala mais compacta e que os itens fossem mais altamente correlacionados entre si, utilizou-se a inclusão de itens que tivessem saturação acima de 0,40. Os dados da análise fatorial exploratória indicaram a possibilidade de fatoração, e os resultados das análises de componentes principais e da rotação varimax revelaram a existência de dois fatores, totalizando 20 itens, explicando $41,36 \%$ da variância. Os coeficientes de consistência interna dos fatores foram calculados empregando os coeficientes de Cronbach. $\mathrm{O}$ alfa de Cronbach do fator 1, que contempla 14 itens, foi de 0,87 ; e do fator 2 , com seis itens, foi de 0,77

\section{Procedimentos}

O estudo foi iniciado após a aprovação da equipe gestora e dos professores das escolas contatadas para a realização da pesquisa. O presente estudo está de acordo com a Resolução n ${ }^{\circ}$ 466/2012 do Conselho Nacional de Saúde, que versa sobre os aspectos éticos da pesquisa com seres humanos. Os pais ou responsáveis pelas crianças foram contatados por meio de um documento escrito, com esclarecimentos sobre a pesquisa, além da solicitação de assinatura no Termo de Consentimento Livre e Esclarecido. A coleta de dados foi realizada nas dependências das escolas, em salas de aula destinadas à realização da pesquisa no mesmo turno das aulas. As crianças responderam aos instrumentos coletivamente, em grupos de 10 alunos, e com duração aproximada de 30 minutos. A leitura de ambas as escalas foi feita pela experimentadora responsável, que lia a primeira questão e aguardava o preenchimento dos alunos, e assim sucessivamente, até o final do instrumento.

\section{Resultados}

\section{Percepção Infantil da Motivação Escolar}

As estatísticas descritivas que avaliam as diferenças entre os anos escolares e a motivação escolar (EAME-IJ) evidenciam que a média dos alunos do $3^{\circ}$, $4^{\circ}$ e $5^{\circ}$ ano relativa à motivação intrínseca foi acima do ponto médio da escala, que é 10 pontos. Na motivação extrínseca, os alunos do $3^{\circ}$ ano obtiveram média acima do ponto médio da escala; entretanto, os alunos do $4^{\circ} \mathrm{e}$ do $5^{\circ}$ ano obtiveram médias abaixo do ponto médio da escala, que é 10 pontos. Esses dados podem ser observados na Tabela 1.

Uma ANOVA foi realizada com o intuito de verificar se havia diferenças na orientação motivacional das crianças nos diferentes anos escolares. As análises indicaram diferenças significativas em função do ano escolar para ambas as fontes de motivação, intrínseca $(F[2,338]=3,317 ; p \leq 0,037)$ e extrínseca $(F[2,338]=$ 7,881; $p \leq 0,001)$.

Na Tabela 2, observa-se que, nos resultados do teste de Tukey, para ambas as fontes de motivação, houve a formação de apenas dois grupos para explicar a variância do escore total entre os anos escolares. Para a motivação intrínseca, houve a formação de dois grupos, contudo, as crianças do $4^{\circ}$ ano ficaram congregadas nos dois grupos, evidenciando as diferenças entre as crianças do $5^{\circ}$ e do $3^{\circ}$ ano. Na motivação extrínseca, formou-se um grupo para as crianças do $5^{\circ}$ e $4^{\circ}$ ano e um segundo grupo para as crianças do $3^{\circ}$ ano, para a motivação extrínseca, mostrando as diferenças entre as crianças do $4^{\circ}$ e do $3^{\circ}$ ano.

\section{Percepção Infantil do Suporte Familiar}

Na Tabela 3, observa-se que a média dos participantes do $3^{\circ}, 4^{\circ}$ e $5^{\circ}$ ano referente ao suporte afetivo ficou acima do ponto médio da escala, que é 21 pontos.

Tabela 1

Estatistica Descritiva da EAME-IJ para Estudantes do $3^{\circ}, 4^{\circ}$ e $5^{\circ}$ Anos do Ensino Fundamental

\begin{tabular}{ccccccc}
\hline & Motivação & $\mathrm{N}$ & Média & Desvio Padrão & Mínimo & Máximo \\
\hline $3^{\circ}$ & Intrínseca & 84 & 17,26 & 2,98 & 4 & 20 \\
& Extrínseca & 84 & 11,99 & 5,55 & 0 & 20 \\
$4^{\circ}$ & Intrínseca & 134 & 16,76 & 2,96 & 9 & 20 \\
& Extrínseca & 134 & 9,93 & 5,75 & 0 & 20 \\
$5^{\circ}$ & Intrínseca & 123 & 16,20 & 2,88 & 8 & 20 \\
& Extrínseca & 123 & 8,94 & 5,01 & 0 & 19 \\
\hline
\end{tabular}


Com relação ao suporte educativo familiar, a média do $3^{\circ}, 4^{\circ}$ e $5^{\circ}$ ano também ficou acima do ponto médio da escala, que é 9 pontos, o mesmo sendo verificado para o suporte material familiar, em que o ponto médio da escala é 5 pontos.

Uma ANOVA foi realizada com o intuito de verificar a existência de diferenças significativas entre as médias das crianças na percepção de suporte familiar em função do ano escolar. Os resultados revelaram que houve diferença estatisticamente significativa somente para o fator suporte educativo familiar $(F[2,339]=$ $8,451 ; p \leq 0,001)$.

De acordo com a Tabela 4, observa-se que houve a formação de apenas dois grupos para explicar a variância do escore total entre os anos escolares para o suporte educativo familiar, um grupo para as crianças do $5^{\circ}$ e $4^{\circ}$ ano e um segundo grupo para as crianças do $3^{\circ}$ ano, ressaltando as diferenças entre as crianças do $4^{\circ}$ e do $3^{\circ}$ ano.

\section{Correlação entre a Percepscão Infantil da Motivação e do Suporte Familiar}

De acordo com a Tabela 5, observa-se correlação positiva, significante e baixa entre a motivação intrínseca e todos os aspectos do suporte familiar, aspecto afetivo, educativo, material e a EPISAF geral, demonstrando que quanto maior a motivação intrínseca dos alunos do Ensino Fundamental maior a percepção infantil do suporte familiar em todos os seus aspectos. Revela, ainda, uma correlação negativa, significante e baixa entre a motivação extrínseca e o aspecto afetivo do suporte familiar $(r=-0,182, p \leq 0,001)$, evidenciando que quanto maior a motivação extrínseca dos alunos menor a percepção infantil do suporte afetivo familiar.

Tabela 2

Teste de Tukey para a EAME-IJ em Função do Ano Escolar

\begin{tabular}{lcccc}
\hline \multirow{2}{*}{ Motivação } & Ano escolar & $\mathrm{N}$ & \multicolumn{2}{c}{ Subgrupo para alfa $=0,05$} \\
\cline { 4 - 5 } Intrínseca & $5^{\circ}$ & 123 & 16,20 & 2 \\
& $4^{\circ}$ & 134 & 16,76 & 16,76 \\
& $3^{\circ}$ & 84 & & 17,26 \\
& $p$ & & 0,34 & 0,420 \\
\hline Extrínseca & $5^{\circ}$ & 123 & 8,94 & 11,99 \\
& $4^{\circ}$ & 134 & 9,93 & 1,00 \\
\hline
\end{tabular}

Tabela 3

Estatística Descritiva da EPIS AF para os Estudantes do $3^{\circ}, 4^{\circ}$ e $5^{\circ}$ Ano do Ensino Fundamental

\begin{tabular}{lcccccc}
\hline Ano escolar & Suporte & $\mathrm{N}$ & Média & Desvio Padrão & Mínimo & Máximo \\
\hline $3^{\circ}$ & Afetivo & 84 & 34,48 & 7,73 & 0 & 42 \\
& Educativo & 84 & 12,17 & 3,93 & 0 & 18 \\
& Material & 84 & 8,55 & 1,39 & 4 & 10 \\
\hline $4^{\circ}$ & Afetivo & 133 & 34,08 & 6,72 & 8 & 42 \\
& Educativo & 134 & 10,57 & 4,63 & 0 & 18 \\
& Material & 134 & 8,31 & 1,56 & 3 & 10 \\
\hline $5^{\circ}$ & Afetivo & 124 & 34,43 & 6,12 & 2 & 42 \\
& Educativo & 124 & 9,62 & 4,40 & 1 & 18 \\
& Material & 123 & 8,28 & 1,44 & 3 & 10 \\
\hline
\end{tabular}


Tabela 4

Teste de Tukey para o Fator Educativo da EPIS AF em Função do Ano Escolar

\begin{tabular}{lccc}
\hline \multirow{2}{*}{ Ano escolar } & $\mathrm{N}$ & \multicolumn{2}{c}{ Subgrupo para alfa $=0,05$} \\
\cline { 3 - 4 } & & 1 & 2 \\
$5^{\circ}$ & 123 & 9,62 & \\
$4^{\circ}$ & 134 & 10,57 & 12,17 \\
$3^{\text {o }}$ & 84 & & 1,00 \\
$P$ & & 0,24 & \\
\hline
\end{tabular}

Tabela 5

Correlação entre a Motivação e o Suporte Familiar

\begin{tabular}{lcccc}
\hline & \multicolumn{2}{c}{ Motivação Intrínseca } & \multicolumn{2}{c}{ Motivação Extrínseca } \\
\cline { 2 - 5 } & $r$ & $p$ & $r$ & $P$ \\
\hline Aspecto afetivo & 0,33 & $<0,001^{* *}$ & $-0,18$ & $0,001^{* *}$ \\
Aspecto educativo & 0,28 & $<0,001^{* *}$ & 0.07 & 0,192 \\
Aspecto material & 0,33 & $<0,001^{* *}$ & $-0,07$ & 0,144 \\
EPISAF geral & 0,37 & $<0,001^{* *}$ & $-0,09$ & 0,073 \\
\hline
\end{tabular}

Nota. $* * p<0,001$

\section{Discussão}

A análise dos dados referentes à motivação revela que as crianças de anos escolares menos avançados tiveram médias mais altas tanto na motivação intrínseca quanto na extrínseca, quando comparadas aos estudantes de anos mais avançados. Esses dados são semelhantes aos estudos de Ferreira, Cardoso e Abrantes (2011); Guidetti (2013); Hornstra, Veen, Peetsma e Volman (2013); Lemos e Veríssimo (2014); Martinelli e Sisto (2010); e Meyersa, Pignaultb e Houssemandc (2013), que afirmam que as crianças de anos iniciais de escolarização apresentam-se mais motivadas que as crianças com mais anos de escolarização.

Visto que, na obtenção de um comportamento motivado interagem as características de personalidade e as características ambientais percebidas pelos indivíduos, acredita-se que, no contexto escolar, esta possa ser modificada por meio de mudanças que dizem respeito ao aluno e ao ambiente de aprendizagem escolar (Ferreira et al., 2011). Segundo a literatura, o declínio na motivação escolar pode ser explicado por diferentes fatores, entre eles os relacionados aos alunos, como metas não relacionadas à aprendizagem (Solis, 2003), desinteresse e falta de pré-requisito para compreender o conteúdo ministrado (Ferreira et al., 2011), e ao professor, enfatizando o tipo de tarefas escolares propostas e a expectativa com relação à aprendizagem de seus alunos (Solis, 2003).

Ainda no que diz respeito à análise das orientações motivacionais intrínseca e extrínseca, os dados deste estudo revelam que, apesar do declínio de ambas, com o avanço da escolaridade, os estudantes se declararam mais motivados intrinsecamente do que extrinsecamente nos anos escolares investigados. Esse resultado é semelhante aos estudos de Ferreira et al. (2011); Gillet, Vallerand e Lafrenière (2012); Lemos e Veríssimo (2014), e Martinelli e Sisto (2010) que verificaram um predomínio da motivação intrínseca sobre a extrínseca nos participantes analisados em seus estudos.

Já os dados da percepção infantil do suporte familiar mostram que os alunos dos anos escolares investigados identificaram um suporte familiar afetivo, educativo e material acima do ponto médio permitido pela escala. Sabendo-se que o ambiente familiar ocupa posição privilegiada nos estudos do desenvolvimento infantil e que a literatura da área busca, cada vez mais, avaliar em que medida as experiências familiares têm efeitos ao longo da vida afetiva, cognitiva e social do indivíduo (Papalia, Olds, \& Feldman, 2010; Santos, 
Martinelli, \& Monteiro, 2012), os dados deste trabalho demonstraram que as crianças entre sete e 13 anos percebem de forma positiva o suporte que seus pais oferecem a elas, nesse período de seu desenvolvimento.

Visto que o presente estudo avalia três aspectos da percepção infantil do suporte familiar, os resultados apontaram que os estudantes declararam, nos três anos investigados, médias muito semelhantes tanto para o suporte afetivo quanto para o suporte material familiar. Por sua vez, com relação ao suporte educativo familiar, evidenciou-se que, conforme o aluno prossegue no seu percurso escolar, diminui sua percepção de suporte educativo familiar recebido. Por outro lado, a literatura tem apontado que o suporte educativo familiar é elemento fundamental na aprendizagem das crianças (Galindo \& Sheldon, 2012; Gonzalez-DeHass, Willems, \& Doan Holbein, 2005; Santos et al., 2012), portanto, é importante refletir em que grau é necessário o suporte educativo familiar para os estudos nesse período do desenvolvimento. Pode-se aventar que à medida que as crianças crescem, os pais aumentem gradativamente o grau de autonomia dado aos filhos, principalmente no que diz respeito aos afazeres, como fazer a lição de casa, arrumar o material escolar, cuidar de seus pertences, seguir a rotina estipulada, e esse fato poderia explicar o declínio da percepção infantil, conforme aumenta a escolaridade, sobre o suporte educativo dado pelos pais.

Para as relações entre o suporte familiar e a motivação escolar, encontrou-se que, quanto maior a motivação intrínseca dos alunos, maior a percepção infantil do suporte familiar, em todos os seus aspectos. Os dados revelaram, ainda, uma correlação negativa entre a motivação extrínseca e o aspecto afetivo do suporte familiar, evidenciando que quanto maior a motivação extrínseca dos alunos, menor a percepção infantil do suporte afetivo familiar. A literatura discorre sobre essa relação (Gonzalez-DeHass et al., 2005; Solis, 2003), entretanto, não foram encontrados estudos empíricos que a tenham investigado. Sabendo-se que o suporte familiar é um dos aspectos necessários tanto para o desenvolvimento quanto para as aprendizagens infantis (Papalia et al., 2010), e que a motivação humana, segundo a TAD (Ryan \& Deci, 2000) focaliza as tendências naturais humanas para o crescimento, desenvolvimento e bem-estar no processo de interação com as condições socioculturais, pode-se inferir que as percepções infantis do suporte familiar atuem na razão das escolhas e do esforço direcionado à aprendizagem.

\section{Considerações Finais}

Na presente pesquisa, a análise da motivação escolar gerou conhecimentos que podem contribuir para ampliar o entendimento dos profissionais da Psicologia e Educação sobre essa variável. Considera-se importante discutir sobre a relevância de manter a motivação intrínseca dos alunos com o progredir da escolaridade e pode-se pensar em novos estudos que investiguem que elementos deveriam estar presentes no ambiente escolar e familiar e que contribuiriam com a manutenção da motivação intrínseca. Assim, avaliar o suporte efetivamente recebido e a motivação escolar intrínseca dos alunos poderia ser uma nova frente de investigação que ampliaria o olhar sobre essas variáveis e suas relações.

No que diz respeito ao suporte familiar educativo, o que se pôde verificar neste estudo foi que as crianças de anos escolares mais avançados perceberam o suporte educativo recebido menos presente do que as crianças mais novas. A respeito desse dado é importante fazer algumas considerações. É importante destacar que a investigação conduzida se pautou na percepção dos alunos (filhos) sobre esse suporte, e não efetivamente sobre sua ocorrência ou não. Assim é importante considerar que os dados não se referem ao suporte familiar efetivamente recebido, mas ao suporte percebido pelos filhos. Embora o dado se refira a uma percepção, e esta pode não traduzir a realidade dos fatos, considera-se que ela pode influenciar os posicionamentos individuais relativos a uma outra realidade. Aponta-se que uma interferência possível pode estar relacionada com a percepção dos filhos sobre o suporte familiar afetivo disponibilizado pelos pais e a motivação relacionada aos estudos. Como até o presente momento esse foi um tema pouco pesquisado, o que se apresenta é uma reflexão sobre quais comportamentos afetivos dos pais poderiam motivar seus filhos para as atividades escolares.

Pontua-se que entre os aspectos de suporte afetivo que podem contribuir com a motivação escolar estejam aqueles relativos ao estimulo da curiosidade e a vontade de aprender, bem como o incentivo e valorização das conquistas infantis. Acredita-se que, entre outros, estes sejam comportamentos que oferecem um suporte positivo e que podem favorecer a motivação escolar intrínseca dessas crianças. Por outro lado, considerando que as demandas infantis por atenção, envolvimento e participação, talvez sejam mais prontamente atendidas quando os filhos apresentam os resultados almejados pelos pais diante dos estudos, pode-se inferir que essas 
crianças que declararam com maior frequência estudar para não ficar de castigo e buscar bons resultados escolares para agradar seus pais agem dessa forma para obter maior atenção dos pais, uma vez que esta possa lhes ser mais direcionada nesses casos. Situações como essas, frequentemente vivenciadas, poderiam estimular uma maior motivação extrínseca das crianças. A reflexão que se coloca é sobre os valores que se pretende estimular nos indivíduos, ou seja, um valor maior aos resultados escolares ou ao estímulo à vontade de saber. Acredita-se que, entre outras, essas questões possam direcionar novas hipóteses investigativas.

\section{Referências}

Aikens, N. L., \& Barbarin, O. (2008). Socioeconomic differences in reading trajectories: The contribution of family, neighborhood, and school contexts. Journal of Educational Psychology, 100(2), 235-251. doi: 10.1037/0022-0663.100.2.235

Andrada, E. G. C. (2007). O Treinamento de suporte parental (TSP) como fator de promoção do suporte parental e do desempenho escolar de crianças na primeira série (Tese de doutorado). Centro de Filosofia e Ciências Humanas. Universidade Federal de Santa Catarina. Florianópolis.

Baptista, M. N. (2005). Desenvolvimento do inventário de percepção de suporte familiar (IPSF): Estudos psicométricos preliminares. Psico-USF, 10(1), 11 19. doi: 0.1590/S1413-82712005000100003

Benetti, S. P. C., \& Balbinotti, M. A. A. (2003). Elaboração e estudo de propriedades psicométricas do inventário de práticas parentais. Psico-USF, 8(2), 103-113. doi: 10.1590/S1413-82712003000200002

Bong, M. (2008). Effects of parent-child relationships and classroom goal structures on motivation, help-seeking avoidance, and cheating. The Journal of Experimental Education, 76(2), 191-217. doi: 10.3200/JEXE.76.2.191-21

Bradley, R. H., McKelvey, L. M., \& Mansell, L. W. (2011). Does the quality of stimulation and support in the home environment moderate the effect of early education programs? Child Development, 82(6), 21102122. doi: $10.1111 / j .1467-8624.2011 .01659 . x$

Cheung, C. S., \& Pomerantz, E. M. (2011). Parents' involvement in children's learning in the United States and China: Implications for children's academic and emotional adjustment. Child Development, 82(3), 932-950. doi: 10.1111/j.1467-8624.2011.01582.x

Cia, P., Pamplin, R. C. O., \& Williams, L. C. A. (2008). O impacto do envolvimento parental no desempenho acadêmico de crianças escolares. Psicologia em Estudo, 13(2), 351-360. doi: 10.1590/ S1413-73722008000200018

Deci, E. L., \& Ryan, R. M. (2008). Self-determination theory: A macrotheory of human motivation, development, and health. Canadian Psychology, 49(1), 182-185. doi: $10.1037 / \mathrm{a} 001280$

Dunst, C. J., \& Leet, H. E. (1986). Family resource scale (FRS). Newton: Brookline Books.

Fantuzzo, J., Tighe, E., McWaayne, C. M., Davis, G., \& Childs, S. (2002). Parent involvement in early childhood education scale (PICES). Journal for Early Intervention Field, 6(1), 3-21. doi: 10.1207/ s19309325nhsa0601_2

Ferreira, M., Cardoso, A. P., \& Abrantes, J. L. (2011). Motivation and relationship of the student with the school as factors involved in the perceived learning. Procedia: Social and Behavioral Sciences, 29(00), 1707-1714. doi: 10.1016/j.sbspro.2011.11.416

Galindo, C., \& Sheldon, S. B. (2012). School and home connections and children's kindergarten achievement gains: The mediating role of family involvement. Early Childhood Research Quarterly, 27(1), 90-103. doi: 10.1016/j.ecresq.2011.05.004

Gillet, N., Vallerand, R. J., \& Lafrenière, M. A. K. (2012). Intrinsic and extrinsic school motivation as a function of age: The mediating role of autonomy support. Social Psychology of Education, 15(1), 77-95. doi: 10.1007/s11218-011-9170-2

Gonzalez-DeHass, A. R., Willems, P. P., \& Doan Holbein, M. F. (2005). Examining the relationship between parental involvement and student motivation. Educational Psychology Review, 17(2), 99-123. doi: $10.1007 /$ s10648-005-3949-7

Guidetti, A. A. (2007). Ambiente familiar e desempenho acadêmico de crianças do ensino fundamental (Dissertação de mestrado). Programa de Pós-Graduação em Educação. Universidade Estadual de Campinas. Campinas.

Guidetti, A. A. (2013). Desempenho escolar e percepscão infantil da motivação e suporte familiar (Tese de doutorado). 
Programa de Pós-Graduação em Educação. Universidade Estadual de Campinas. Campinas.

Guidetti, A. A. \& Martinelli, S. C. M. (2009). Desempenho em leitura e suas relações com o contexto familiar. Em A. A. A. Santos, E. Boruchovitch e K. L. Oliveira (Eds.). Cloze: Um instrumento de diagnóstico e intervenção (pp. 283-309). São Paulo: Casa do Psicólogo.

Guidetti, A. A. \& Martinelli, S. C. M. (2010). Escala da percepscão infantil dos suportes do ambiente familiar - EPISAF. Universidade Estadual de Campinas. Manuscrito não submetido para publicação.

Horn, M. L. V., Jaki, T., Masyn, K, Ramey, S. L., Smith, J. A., \& Antaramian, S. (2009). Assessing differential effects: Applying regression mixture models to identify variations in the influence of family resources on academic achievement. Developmental Psychology, 45(5), 1298-1313. doi: 10.1037/a0016427

Hornstra, L., Veen, V. D. I., Peetsma, T., \& Volman, M. (2013). Developments in motivation and achievement during primary school: A longitudinal study on group-specific differences. Learning and Individual Differences, 23(1), 195-204. doi: 10.1016/j. lindif.2012.09.004

Iachini, A. L., Buettner, C., Anderson, B. D., \& Reno, R. (2013). Exploring students' perceptions of academic disengagement and reengagement in a dropout recovery charter school setting. Children \& Schools, 35(2), 113-120. doi: 10.1093/cs/cdt005

Lemos, M. S., \& Veríssimo, L. (2014). The relationships between intrinsic motivation, extrinsic motivation, and achievement, along elementary school. Procedia: Social and Behavioral Sciences, 112(00), 930-938. doi: 10.1016/j.sbspro.2014.01.1251

Martinelli, S. C., \& Sisto, F. F. (2010). Motivação de estudantes: Um estudo com crianças do ensino fundamental. Avaliação Psicológica, 9(3), 413-420. Recuperado de http://pepsic. bvsalud.org/scielo.php?script=sci_arttext\&pi$\mathrm{d}=$ S1677-04712010000300008\&lng=pt\&tlng=pt.

Martinelli, S. C., \& Sisto, F. F. (2011). Escala para avaliação da motivação escolar infanto-juvenil (EAME-IJ). São Paulo: Casa do Psicólogo.

Marturano, E. M. (2006). O inventário de recursos do ambiente familiar. Psicologia: Reflexão e Crítica, 19(3), 498-506. doi: 10.1590/S0102-79722006000300019
Meyersa, R., Pignaultb, A., \& Houssemandc, C. (2013). The role of motivation and self-regulation in dropping out of school. Procedia: Social and Behavioral Sciences, 89(00), 270-275. doi: 10.1016/j. sbspro.2013.08.845

Monteiro, R. M (2010). Motivação e recursos familiares: relação com a compreensão de leitura em escolares. (Dissertação de Mestrado). Universidade São Francisco - Programa de Pós-Graduação Stricto Sensu em Psicologia.

Moos, R., \& Moos, B. (1994). Family environment scale manual: Development, applications, research. Palo Alto: Consulting Psychologists Press.

Moreira, P. A. S., Dias, P., Vaz, F. M., \& Vaz, J. M. (2013). Predictors of academic performance and school engagement integrating persistence, motivation and study skills perspectives using person-centered and variable-centered approaches. Learning and Individual Differences, 24(2), 117-125. doi: 10.1016/j. lindif.2012.10.016

Papalia, E. D., Olds, S. W., \& Feldman, R. D. (2010). Desenvolvimento bumano. Porto Alegre: ARTMED.

Polonia, A. C., \& Dessen, M. A. (2005). Em busca de uma compreensão das relações entre família e escola. Psicologia Escolar e Educacional, 9(2), 303-312. doi: 10.1590/S1413-85572005000200012

Próspero, M., Russell, A. C., \& Gupta, S. V. (2012). Effects of motivation on educational attainment: Ethnic and developmental differences among first-generation students. Journal of Hispanic Higher Education, 11(1), 100-119. doi: 10.1177/1538192711435556

Ryan, R. M., \& Deci, E. L. (2000). Intrinsic and extrinsic motivations: Classic definitions and new directions. Contemporary Educational Psychology, 25(1), 54-67. doi: 10.1006/ceps.1999.1020

Ryan, R. M., Lynch, M. F., Vansteenkiste, M., \& Deci, E. L. (2011). Motivation and autonomy in counseling, psychotherapy, and behavior change: A look at theory and practice. The Counseling Psychologist, 39(2), 193-260. doi: 10.1177/0011000009359313

Santos, A. A. A., Martinelli, S. C., \& Monteiro, R. M. (2012). Suportes e recursos familiares: Relações com o contexto escolar. Em M. N. Baptista \& M. L. M. Teodoro (Eds). Psicologia de Familia: teoria, avaliação e intervenção. (pp.137-144). Porto Alegre: ARTMED. 
Solis, M. P. (2003). Orientación educativa. Madrid: Thomson.

Spera, C. (2006). Adolescents' perceptions of parental goals, practices and styles in relation to their motivation and achievement. The Journal of Early Adolescence, 26(4), 456-490. doi: 10.1177/0272431606291940

Sternberg, R. J. (2010). Psicologia cognitiva. São Paulo: Cengage Learning.

Willems, P. P., \& Gonzalez-DeHass, A. R. (2012). School-community partnerships: Using authentic contexts to academically motivate students. School Community Journal, 22(2), 9-30. doi: 84446398

Yune, T. (2014). Addressing reciprocity between families and schools: Why these bridges are instrumental for students academic success. Improving Schools, 17(1), 18-29. doi: 10.1177/1365480213515296

Recebido em: 08/09/2015

Reformulado em: 30/03/2016; 16/06/2016

Aprovado em: 11/08/2016

Sobre as autoras:

Andréia Arruda Guidetti é doutora em Educação pela Universidade Estadual de Campinas e professora titular do curso de Psicologia da Universidade Paulista.

E-mail:deia.arruda@outlook.com.br

Selma de Cássia Martinelli é professora livre docente do Departamento de Psicologia Educacional da UNICAMP/ SP e Líder do Grupo de Estudos e Pesquisa em Psicopedagogia (GEPESP).

E-mail:selmacm@unicamp.br

Contato com as autoras:

Av. Bertrand Russel, 801

Cidade Universitária Zeferino Vaz

Campinas-SP, Brasil

CEP: 13083-865

Psico-USF, Bragança Paulista, v. 22, n. 3, p. 515-525, set./ dez. 2017 
